\title{
Evolutionary consequences of a search image
}

\author{
E. van Leeuwen ${ }^{\mathrm{a}, *}$, V.A.A. Jansen ${ }^{\mathrm{b}}$ \\ ${ }^{a}$ Centre for Ecological and Evolutionary Studies, University of Groningen, PO Box \\ 14, 9750 AA Haren, The Netherlands \\ ${ }^{\mathrm{b}}$ School of Biological Sciences, Royal Holloway, University of London, Egham, \\ Surrey TW20 OEX, UK
}

\begin{abstract}
Many predators are able to become better at spotting cryptic prey by recognising specific clues, but by concentrating on one prey type they will become worse at spotting other prey types. This phenomenon is known as the formation of a search image for a certain prey by a predator and is related to apostatic selection. Here we study the evolution of a search image in the predator by formulating and analysing a mathematical model. The predator forages for two prey types and is able to form an independent search image for both prey. The results show that the evolutionary dynamics can be divided into two parts, a fast and a slow part. At first selection pressure will be strong towards a stable ratio of prey, which is the same as the ratio found for the unbeatable prey choice for predators with a Holling type II functional response. Following this the slow dynamics will keep this ratio constant independent of the trait values, but the predator will slowly evolve towards a stronger search image and ultimately become a specialist predator or slowly evolve towards generalist with a weak search image. In conclusion, the formation of a search image causes the predator to control the prey densities such that the ratio of available prey is kept constant by the predator.
\end{abstract}

Key words: apostatic selection, ideal free forager, predator-prey, specialist, generalist, functional response, population dynamics, search image, switching, searching image

\footnotetext{
* Corresponding author

Email addresses: E.van.Leeuwen@rug.nl (E. van Leeuwen), Vincent. Jansen@rhul.ac.uk (V.A.A. Jansen).
} 


\section{Introduction}

Many prey species rely on hiding or camouflage to keep from being captured. As a result, prey species are often difficult to spot for predators, but predators can enhance their capture rate by looking for specific clues of a certain prey type. This is called the formation of a search image by a predator (Tinbergen, 1960) and is related to apostatic selection (Bond, 2007). Research shows that a predator can only form a search image for one prey type at a time and this causes other prey types to be detected less frequently (Pietrewicz and Kamil, 1981; Bond, 1983; Gendron, 1986; Bond, 2007).

Although the formation of a search image has historically been associated with bird species (Tinbergen, 1960; Pietrewicz and Kamil, 1979; Bond, 1983; Allen et al., 1998; Bond and Kamil, 2006), there is a strong indication that predators from different classes form search images. For example, Jackson and Li (2004) showed that hunting spiders also form search images. This result is especially surprising in that search images are mostly associated with vertebrate species, which have complex brain functions (Dukas and Kamil, 2001). Melcer and Chiszar (1989) also found an indication of a search image being formed in snakes, using a chemical signature rather than visual clues.

The formation of a search image can have an important effect on population dynamics. If we make the reasonable assumption that a search image would be formed for the more abundant prey type, this leads to a disproportionally high predation rate on the more abundant prey type and, thus, to prey switching (Murdoch, 1969; Oaten and Murdoch, 1975; Van Leeuwen et al., 2007). Search images cause prey switching by a predator, which leads to coexistence between prey species even if the prey species are in direct competition (Murdoch, 1969), such behaviour could also lead to polymorphism in prey species (Allen et al., 1998; Bond and Kamil, 2006).

While many predators are able to form a search image, it seems that there is a trade off. When the predator forms a search image for a certain prey this means that it becomes worse at spotting other prey types (Dawkins, 1971; Pietrewicz and Kamil, 1979; Reid and Shettleworth, 1992; Bond and Kamil, 1999). It has been theorised that this trade off is caused by limited computational resources of the predator (Dukas and Kamil, 2001; Dukas, 2002; Bond, 2007). This indicates that the trade off is a fixed cognitive constraint and selection will not change this trade off. The existence of this trade off implies that a predator would benefit from forming a stronger search image through increased taking of one prey type, yet would suffer through decreased feeding on alternative prey. The strength of the search image the predator forms will, therefore, influence its fitness and be under selective pressure. 
Analysis of the evolution of predators in one predator-two prey systems where the predator is unable to form a search image, shows that in such a system the predator will evolve its prey diet towards a state which is the well mixed equivalent of an Ideal Free Distribution (IFD; Fretwell and Lucas, 1970; Kruvan et al., 2008), in that predators regulate their prey in such a way that no predator can gain from having a different diet (Abrams, 2006b,a; Schreiber and Tobiason, 2003; Rueffler et al., 2006, 2007). Well mixed here refers to the fact that the prey are mixed together instead of distributed among patches. One important difference to the IFD is that the gain of the predator need not be equal for both prey, particularly so if the predators have a Holling type II functional response. We will refer to a predator that has a type II functional response and that cannot improve its diet by chosing a different prey as an Ideal Free Forager (IFF). By including the search image into the simpler model we can gain insight into how robust the previous findings, and specifically the IFF, are when more complicated behaviour is considered.

Search images and their long term effect on prey species have received much attention as a possible cause for polymorphism in prey species (Allen, 1988; Bond and Kamil, 2002, 2006; Ruxton et al., 2007). In contrast, the question why predators form search images has received very little attention. In this paper we try to answer this question with a theoretical model. For this we use a one predator-two prey model based on a previously developed functional response in which attack rates depend on the last encountered prey (Oaten and Murdoch, 1975; Van Leeuwen et al., 2007) and include the aforementioned trade off. We then use this model for the evolution of search images for two prey species. The model shows under which circumstances search images can evolve and what the effect is on relative prey densities. The resulting evolutionary dynamics can be split into a fast and a slow part. First, selection pressure drives the dynamics towards a state in which the predator is an IFF. Once the predator is an IFF the ratio of prey densities is kept constant by the predator, but the actual prey densities can still be subject to selection. At that point slow evolution towards specialisation or generalisation, i.e. very strong search image or no search image, will occur.

\section{Theory and methods}

\subsection{Introducing the model}

We formulate a mathematical model for a predator which feeds on two prey types. The predator is able to form an independent search image for each prey. We assume that a predator's attack rate depends on the previous prey it has encountered. Therefore, to describe a predator feeding on 2 prey types 
we need 4 different attack rates, which we will denote as $a_{i j}$, where $i$ is the prey type currently attacked and $j$ is the previous prey type attacked. This means that we assume that the search image is formed after an encounter with a certain prey: a predator that just attacked a prey of type 1 will form a search image for prey type 1 and therefore get an higher attack rate on this prey type. However, because it is now specifically looking for prey type 1 it will actually get an lower attack rate for the other prey type (Dukas, 2002). This means that we can define the trade off in the attack rates as follows:

$$
\begin{array}{r}
a_{i i}\left(\theta_{i}\right)=a_{i} \theta_{i}^{z} \\
a_{j i}\left(\theta_{i}\right)=a_{j}\left(1-\theta_{i}\right)^{z}
\end{array}
$$

where $i$ and $j$ are 1 or 2 and $i \neq j^{1}, a_{i i}$ the attack rate of the predator on prey type $i$ if it has formed a search image for prey type $i$, i.e. its previous prey was of type $i, a_{j i}$ the attack rate on prey type $j$ when the predator has formed a search image for prey type $i, \theta_{i}$ is the value of the trait which determines the strength of its search image for prey type $i, a_{i}$ the base attack rate of the predator on prey type $i$ and $z$ a trade off coefficient (following Levins, 1962) where $z=1$ is a linear trade off, $z<1$ a concave trade off and $z>1$ indicates a convex trade off. In what follows we will leave out dependence of parameters on traits, i.e. $a_{i i}\left(\theta_{i}\right)$ is shortened to $a_{i i}$ if the meaning is unambiguous, to improve readability. High values of $\theta_{i}$ indicate a strong search image, while lower values mean a weaker search image. Note that if $\theta_{i}<a_{j}^{\frac{1}{z}} /\left(a_{1}^{\frac{1}{z}}+a_{2}^{\frac{1}{z}}\right)$ the predator will be better at capturing a different prey species than at finding the same again. This would imply a negative search image.

We will use a functional response that uses these four attack rates, and thus include our search image into a population dynamical model. For this we use the functional response first described by Oaten and Murdoch (1975) and extended by Van Leeuwen et al. (2007) that incorporates dietary history in the functional response.

$$
\begin{aligned}
& f_{i}(\theta, N)= \\
& \frac{a_{i j} N_{i}\left(a_{i i} N_{i}+a_{j i} N_{j}\right)}{a_{12} N_{1}+a_{11} a_{12} T_{11} N_{1}^{2}+a_{12} a_{21} N_{1} N_{2}\left(T_{12}+T_{21}\right)+a_{22} a_{21} T_{22} N_{2}^{2}+a_{21} N_{2}}
\end{aligned}
$$

with $\theta=\left(\theta_{1}, \theta_{2}\right)^{T}$ and $N=\left(N_{1}, N_{2}\right)^{T} . N_{k}$ is the density of prey type $k$ and $T_{k l}$ the handling time for prey type $k$ when $l$ was the previous prey encountered.

The population dynamics are based on a Rosenzweig-MacArthur model (Rosen-

$\overline{1}$ The condition $i \neq j$ is implicitly assumed throughout this manuscript 
zweig and MacArthur, 1963) for two prey species:

$$
\begin{array}{r}
\frac{\mathrm{d} P}{\mathrm{~d} t}=\left(c_{1} f_{1}(\theta, N)+c_{2} f_{2}(\theta, N)-m\right) P \\
\frac{\mathrm{d} N_{i}}{\mathrm{~d} t}=N_{i} g_{i}\left(N_{i}, N_{j}\right)-f_{i}(\theta, N) P
\end{array}
$$

with $c_{i}$ the conversion factor of prey type $i$ into predator and $m$ the mortality rate of the predator. We assume Lotka-Volterra competition between the prey species: $g_{i}\left(N_{i}, N_{j}\right)=r_{i}\left(1-\left(N_{i}+\alpha_{i j} N_{j}\right) / K\right)$ in which, $K$ is the carrying capacity and $\alpha_{i j}$ is the competition effect of prey type $j$ on prey type $i$.

Following Metz et al. (1992) and Geritz et al. (2004), we define fitness as the per capita growth rate of a rare mutant in a background of an abundant resident predator population. Although this fitness extends to non-equilibrum dynamics, we will assume for ease of analysis, that the resident population is in equilibrium. In Van Leeuwen et al. (2007) the population dynamics of this system are fully explored. Under the assumption of neutral competition $\left(\alpha_{i j}=\right.$ $\alpha_{j i}=1$ ), the two prey species will coexist as long as the predator is present and $a_{i i}>a_{j i}$. Under those conditions the system can show a single stable equilibrium or a limit cycle. In the rest of this manuscript we assume that the system is in the stable coexistence equilibrium unless otherwise specified. The per capita growth rate of the predator depends on the number of prey they consume, which, in turn, is determined by the functional response and the mortality rate $(m)$ of the predator. The fitness $(W)$ is thus given by:

$$
\begin{aligned}
& W\left(\theta^{\prime}, \theta\right)=\frac{1}{P} \frac{\mathrm{d} P}{\mathrm{~d} t}= \\
& c_{1} f_{1}\left(\theta^{\prime}, N^{*}(\theta)\right)+c_{2} f_{2}\left(\theta^{\prime}, N^{*}(\theta)\right)-m
\end{aligned}
$$

where $\theta$ is a vector of the resident's trait values, $\theta^{\prime}$ are the trait values of the mutant and $N^{*}(\theta)$ is a vector containing the equilibrium prey densities, which depend on the resident trait values. At equilibrium densities $\left(N^{*}(\theta)\right)$ the fitness (per capita growth rate) of a predator with resident trait values is zero. Therefore, if $W\left(\theta^{\prime}, \theta\right)$ is greater than zero the mutant can invade and possibly replace the predator population, otherwise the mutant will disappear.

To predict the direction of selection pressure we need to solve the partial derivatives of the fitness function to the mutated traits. The selection gradient is:

$$
\nabla W(\theta)=\left(\left.\frac{\partial W\left(\theta^{\prime}, \theta\right)}{\partial \theta_{1}^{\prime}}\right|_{\theta^{\prime}=\theta},\left.\frac{\partial W\left(\theta^{\prime}, \theta\right)}{\partial \theta_{2}^{\prime}}\right|_{\theta^{\prime}=\theta}\right)^{T}
$$

The system has an evolutionary singular strategy when the selection gradient is zero, i.e. at the point where both elements of $\nabla W(\theta)$ are zero. Finally, to model the change of the vector containing the trait values $\left(\theta=\left(\theta_{1}, \theta_{2}\right)^{T}\right)$ over time, which depends on the selection gradient, we use the canonical equation 
as defined by Dieckmann and Law (1996):

$$
\frac{\mathrm{d} \theta}{\mathrm{d} t}=\frac{1}{2} \mu P^{*}(\theta) \sigma^{2} \nabla W(\theta)
$$

with $\mu$ the mutation rate, $\sigma^{2}$ the variance-covariance matrix of the mutation distribution and $P^{*}(\theta)$ the equilibrium predator density as dependent on the resident trait values. The factor of a half results from the details of the derivation and relates to the variance of the lifetime offspring production, which scales the rate of the evolutionary porcess (Durinx et al., 2008). We assume that the mutation rate is independent of the trait value, and is the same for trait 1 as for trait 2. Furthermore, we assume that mutations in $\theta_{1}$ and $\theta_{2}$ are independent and that therefore the off-diagonal elements of the variancecovariance matrix are zero. Since both traits are very similar, i.e. they both concern search images, just for different prey species, we can reasonably assume that the diagonal elements of the matrix take the same value. Under these assumptions $\mu$ and $\sigma^{2}$ only influence the speed of evolutionary change. This allows us to scale time in such as way that $\mu \sigma^{2}=I$, with $I$ the identity matrix. Furthermore, these assumptions do not influence the values at which singular strategies exist, but they might influence stability of the singular strategy.

\subsection{Evolutionary singular strategy}

The equilibrium prey densities are independent of the mutant trait value, because the density of the number of mutant predators is negligible compared to the density of predators with the resident trait value. We can replace one of the prey densities with a constant times the other prey density in the gradient function, i.e. $\rho=N_{2} / N_{1}$, where $\rho$ is the prey ratio. This allows us to rewrite the functional response to one resembling a type II functional response:

$$
\begin{aligned}
& f_{i}(\theta, N)= \\
& \frac{A_{i} N_{i}}{1+A_{1} N_{1} T_{11}+A_{2} N_{2} T_{22}+\Delta T \frac{a_{21} a_{12} N_{1} N_{2}}{a_{12} N_{1}+a_{21} N_{2}}}
\end{aligned}
$$

with

$$
\begin{aligned}
& A_{1}=\frac{a_{12}\left(a_{11}+a_{21} \rho\right)}{a_{12}+a_{21} \rho} \\
& A_{2}=\frac{a_{21}\left(a_{22} \rho+a_{12}\right)}{a_{12}+a_{21} \rho}
\end{aligned}
$$

and $\Delta T=T_{12}+T_{21}-T_{11}-T_{22}$, which is a measurement for the cost in handling time to switching, i.e. if handling prey after switching from one prey to another takes longer (e.g. due to experience) $\Delta T>0$. Now for a rare mutant 
the prey ratio $\rho$ is controlled by the residents that dominate the population. In Van Leeuwen et al. (2007) it was shown that at equilibrium the predator regulate the prey to a fixed ratio which depends on all four attack rates (i.e. $N_{2} / N_{1}=a_{12}\left(a_{11}-a_{21}\right) /\left(a_{21}\left(a_{22}-a_{12}\right)\right)^{2}$. Therefore, the compound attack rates $\left(A_{i}\right)$ depend on both the mutant trait value and the resident trait value.

In appendix $\mathrm{A}$ we show that if we set $\Delta T=0$ the partial derivatives of the fitness function to $\theta_{i}^{\prime}$ are zero when the ratio of prey densities is:

$$
\frac{N_{1}^{*}}{N_{2}^{*}}=-\left.\frac{\partial A_{2} / \partial \theta_{i}^{\prime}}{\partial A_{1} / \partial \theta_{i}^{\prime}}\right|_{\theta^{\prime}=\theta} \frac{\left(c_{2}-m T_{22}\right)}{\left(c_{1}-m T_{11}\right)}
$$

From this it follows that for both elements of $\nabla W$ (eq. 1) to be zero at the same time the following must hold:

$$
-\left.\frac{\partial A_{1} / \partial \theta_{1}^{\prime}}{\partial A_{2} / \partial \theta_{1}^{\prime}}\right|_{\theta^{\prime}=\theta}=-\left.\frac{\partial A_{1} / \partial \theta_{2}^{\prime}}{\partial A_{2} / \partial \theta_{2}^{\prime}}\right|_{\theta^{\prime}=\theta}
$$

If all trade offs are linear $(z=1)$, the solution for both sides of the previous equation is $a_{1} / a_{2}$ and the condition holds. Thus, the prey ratio $(\rho)$ given by eq. (3) is $a_{2}\left(c_{2}-m T_{22}\right) /\left(a_{1}\left(c_{1}-m T_{11}\right)\right)$, the compound attack rates $\left(A_{i}\right)$ are independent of the resident trait value and $\partial A_{i} /\left.\partial \theta_{i}^{\prime}\right|_{\theta^{\prime}=\theta}$ is equal to $\partial A_{i} / \partial \theta_{i}$. Under these conditions the functional response is identical to a type II functional response. Furthermore, at this prey ratio there is an evolutionary singular strategy, because the selection gradient is zero. We can substitute the previous result into the predator growth equation and find that $N_{i}=m /\left(a_{i}\left(c_{i}-m T_{i i}\right)\right)$. Note that for the prey densities to be positive $c_{i}$ has to be greater than $m T_{i i}$.

Substituting our population dynamical solution for the prey densities into eq. (3) and solving to $\theta_{2}$ results in a function $\left(\theta_{2}=h\left(\theta_{1}\right)\right)$ which gives all the different trait values at which there is a singular strategy. Figure 1 shows the resulting function. At this solution the prey ratio is independent of the resident trait values and the compound attack rates $\left(A_{i}\right)$ only depend on the invading trait value. Thus, the system with $\Delta T=0$ and $z=1$ is identical to a type II one predator-two prey system and leads to an Ideal Free Forager (IFF), that controls the prey densities at a specific ratio. We show in the appendix $\mathrm{B}$ that the IFF is a convergence stable equilibrium for this dynamical system. At the IFF the second derivatives to $\theta^{\prime}$ are equal to zero, such that the IFF is at the cusp of giving rise to polymorphism in the predator population. In the analysis for the more complicated cases $(\Delta T \neq 0$ or $z \neq 1)$ this solution will still play an important role in explaining the dynamics.

${ }^{2}$ Under the assumption of neutral competition between the prey, i.e. the prey are equal competitors for the resource. Note that the attack rates here are the attack rates of the resident predator. This ratio also holds when the population dynamics equilibrium is not stable, but in a limit cycle (Van Leeuwen et al., 2007). 


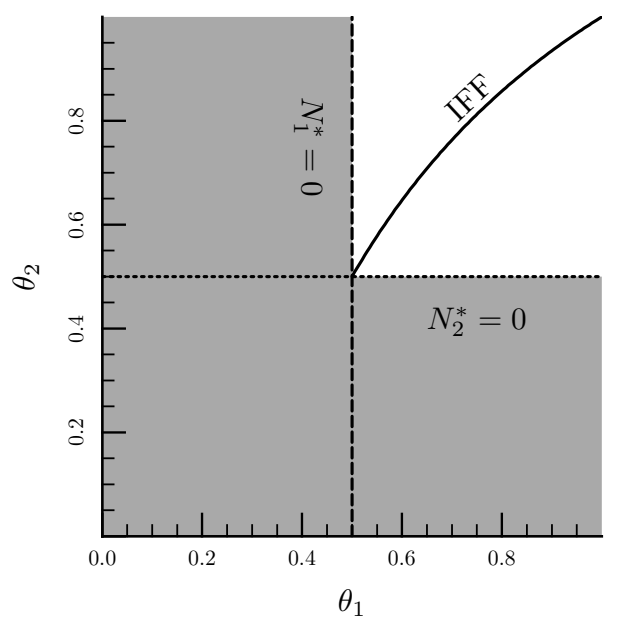

Fig. 1. The singular strategy as a function of $\theta_{2}$ versus $\theta_{1}$. A predator at the singular strategy is known as an Ideal Free Forager (IFF). The grey area shows the parameter values where the coexistence equilibrium is unstable. Parameter values: $a_{1}=a_{2}=1 ; c_{1}=c_{2}=0.15 ; m=0.2 ; T_{11}=0.45 ; T_{22}=0.25$.

The IFF-curve $\left(\theta_{2}=h\left(\theta_{1}\right)\right)$ is biologically valid between the trait values: $\theta_{H}=(1,1)^{T}$ and

$$
\theta_{L}=\left(\frac{a_{2}^{\frac{1}{z}}}{a_{1}^{\frac{1}{z}}+a_{2}^{\frac{1}{z}}}, \frac{a_{1}^{\frac{1}{z}}}{a_{1}^{\frac{1}{z}}+a_{2}^{\frac{1}{z}}}\right)^{T}
$$

Trait values higher than 1 result in negative attack rates. For trait values lower than $\theta_{L} a_{i i}$ is smaller than $a_{j i}$ and, under the assumption of neutral competition between the prey species, the population dynamical equilibrium with both prey species present loses stability and the system shifts to another equilibrium with only one prey species present, i.e. one prey species goes extinct (Van Leeuwen et al., 2007).

\subsection{Cost in handling times}

Behaviour of the evolutionary system is more complicated when $\Delta T$ is not equal to zero, i.e. when there is a handling time cost or benefit when switching from one prey to another. For the following analysis we still set both trade offs to linear $(z=1)$. The singular strategy now obeys (see appendix A):

$$
\begin{aligned}
& \left.\frac{\partial A_{1}}{\partial \theta_{i}^{\prime}}\right|_{\theta^{\prime}=\theta} N_{1}^{*}\left(c_{1}-m T_{11}\right)+\left.\frac{\partial A_{2}}{\partial \theta_{i}^{\prime}}\right|_{\theta^{\prime}=\theta} N_{2}^{*}\left(c_{2}-m T_{22}\right) \\
& -m N_{1}^{*} N_{2}^{*} \Delta T \frac{\mathrm{d} a_{j i}}{\mathrm{~d} \theta_{i}} \frac{a_{i j}^{2} N_{i}^{*}}{\left(a_{12} N_{1}^{*}+a_{21} N_{2}^{*}\right)^{2}}=0
\end{aligned}
$$


It can be seen that $\theta_{H}$ is a singular strategy, because for $i=1$ the term with $\Delta T$ is zero if $a_{12}=0$ and similarly for $i=2$ the third term is zero if $a_{21}=0$.

To study how a non-zero value for $\Delta T$ influences evolutionary dynamics we expand the selection gradient around $\Delta T=0$. The selection gradient for small values of $\Delta T$ can then be approximated by

$$
\nabla W \approx\left(t_{1}(0)+\Delta T t_{1}^{\prime}(0), t_{2}(0)+\Delta T t_{2}^{\prime}(0)\right)^{T}
$$

with $t_{i}(0)$ and $t_{i}^{\prime}(0)$ the first two terms of the Taylor expansion:

$$
\begin{aligned}
& t_{i}(0)= \\
& \frac{\frac{\partial A_{1}}{\partial \theta_{i}} N_{1}^{*}\left(c_{1}-m T_{11}\right)+\frac{\partial A_{2}}{\partial \theta_{i}} N_{2}^{*}\left(c_{2}-m T_{22}\right)}{1+A_{1} N_{1}^{*} T_{11}+A_{2} N_{2}^{*} T_{22}} \\
& t_{i}^{\prime}(0)=\left.\frac{\mathrm{d} t_{i}(\Delta T)}{\mathrm{d} \Delta T}\right|_{\Delta T=0}= \\
& \frac{-m N_{1}^{*} N_{2}^{*} \frac{\mathrm{d} a_{j i}}{\mathrm{~d} \theta_{i}} \frac{a_{i j}^{2} N_{i}^{*}}{\left(a_{12} N_{1}^{*}+a_{21} N_{2}^{*}\right)^{2}}-t_{i}(0) N_{1}^{*} N_{2}^{*} \frac{a_{12} a_{21}}{a_{12} N_{1}^{*}+a_{21} N_{2}^{*}}}{1+A_{1} N_{1}^{*} T_{11}+A_{2} N_{2}^{*} T_{22}}
\end{aligned}
$$

with

$$
t_{i}(\Delta T)=\left.\frac{\mathrm{d}}{\mathrm{d} \Delta T} \frac{\partial W\left(\theta^{\prime}, \theta\right)}{\partial \theta_{i}^{\prime}}\right|_{\theta^{\prime}=\theta}
$$

When $\Delta T$ is small evolutionary change will be dominated by $t_{i}(0)$. As shown in the previous section $t_{i}(0)$ is zero at the IFF and selection pressure will be defined by $\Delta T t_{i}^{\prime}(0)$. The direction of change at the IFF is, thus, defined by the sign of $\Delta T t_{i}^{\prime}(0)$. Simplification then shows us that the direction is equal to the sign of $\Delta T$, since $-\mathrm{d} a_{j i} / \mathrm{d} \theta_{i}$ is positive. This causes the evolutionary dynamics to operate at two time scales and there will be strong selection pressure towards IFF and on the IFF the pressure will be towards lower trait values if $\Delta T$ is negative and towards larger trait values if $\Delta T$ is positive.

Figure 2 illustrates the evolutionary dynamics. The figure was produced by numerically solving the population dynamics to equilibrium. Then numerically evaluating eq. (2) for a short time then solving the population dynamics again, and iterating this procedure. Figures $2 \mathrm{a}$ and $2 \mathrm{~d}$ show the dynamics when $\Delta T$ is zero and the solution quickly approaches the IFF. When $\Delta T$ is positive, the solution quickly approaches the IFF and then slowly evolves towards higher trait values with as evolutionary endpoint the strategy at $\theta_{H}$. At the IFF the prey ratio is kept constant, but the predator is still switching between both prey types. Switching is costly at positive values of $\Delta T$, because the predator experiences longer handling times when switching. Thus, for a positive $\Delta T$ the predator, once it has reached IFF, slowly evolves to switch less (lower values of $a_{i j}$ ) between the two prey species and ultimately becomes a specialist (stops switching) (figs. $2 \mathrm{~b}$ and $2 \mathrm{e}$ ). Similarly, a negative $\Delta T$ will first cause fast evolution towards the IFF and then slow evolution towards lower trait values, 


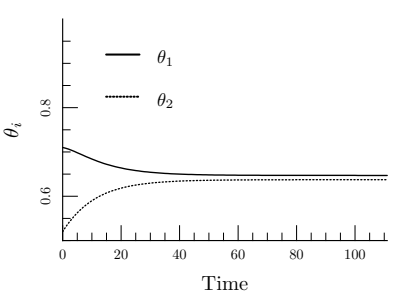

(a)

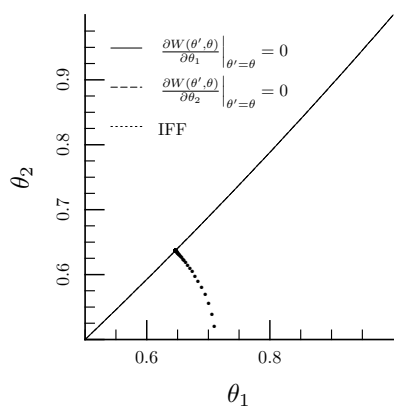

(d)

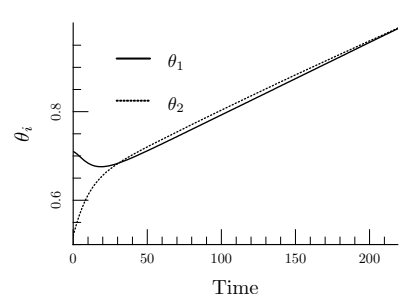

(b)

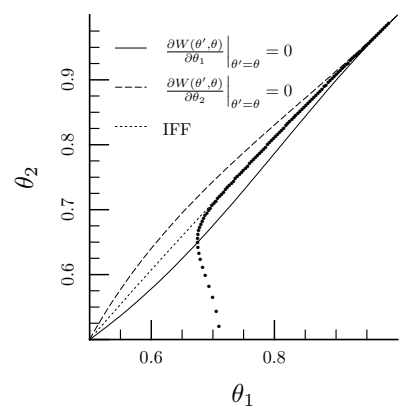

(e)

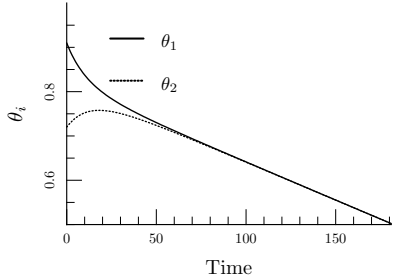

(c)

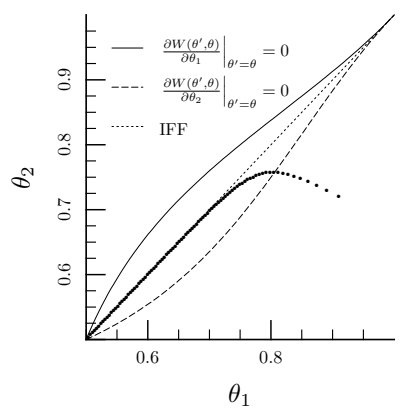

(f)

Fig. 2. The change in trait values as a result of evolutionary dynamics. Showing fast dynamics towards the Ideal Free Forager solution (IFF) and slow dynamics on the IFF. Figures (a)-(c) show the change of the trait values over time. Figures (d)-(f) depict the same results for trait 2 versus trait 1 . The distance between each bullet is equivalent to a constant time step, i.e. the larger the distance the faster evolutionary change. The thin, dotted line is the IFF, while the other lines depict the solution for which the selection gradient in one direction is zero. (a) and (d) show the results when $\Delta T=0$, i.e. $T_{11}=0.2 ; T_{22}=0.25 ; T_{12}=0.2 ; T_{21}=0.25 ;(\mathrm{b})$ and (e) the results for $\Delta T>0$, i.e. $T_{11}=0.2 ; T_{22}=0.15 ; T_{12}=0.45 ; T_{21}=0.45$; (c) and (f) the results $\Delta T<0$, i.e. $T_{11}=0.45 ; T_{22}=0.45 ; T_{12}=0.2 ; T_{21}=0.15$. Parameter values: $a_{1}=a_{2}=1 ; c_{1}=c_{2}=0.15 ; m=0.2 ; r=2.5 ; K=10$.

because switching is actually beneficial due to shorter handling times when switching (figs. 2c and $2 \mathrm{f}$ ). When the trait values reach $\theta_{L}$ the population dynamical equilibrium will lose stability and one of the prey will be driven to extinction.

\subsection{Non linear trade offs}

Next we study the situation where $\Delta T$ is zero, but the trade offs are not linear $(z \neq 1)$. Just like under the previous assumptions the point $\theta=(1,1)^{T}$ is always a singular strategy, regardless of linearity of the trade offs, because the condition given by eq. (4) always holds. To prove this we rewrite our system to polar coordinates, where $\phi$ is the angle in point $\theta_{1}=\theta_{2}$ and $r$ is the distance to this point. Then we take the limit of $r$ to zero. Using this it can 
be shown that:

$$
\lim _{r \rightarrow 0}-\left.\frac{\partial A_{1} / \partial \theta_{i}^{\prime}}{\partial A_{2} / \partial \theta_{i}^{\prime}}\right|_{\theta_{1}^{\prime}=1-r \sin (\phi) ; \theta_{2}^{\prime}=1-r \cos (\phi)}=\frac{a_{1}}{a_{2}}
$$

which proves that eq. (4) holds in this point and that $\theta=(1,1)^{T}$ is a singular strategy.

We can expand the selection pressure equation around linear trade offs $(z=$ $1+\epsilon)$ and show that as long as $\epsilon$ is small evolutionary dynamics will approach the IFF quickly. On the IFF dynamics can be approximated with:

$$
\left.\frac{\mathrm{d} t_{i}(\epsilon)}{\mathrm{d} \epsilon}\right|_{\epsilon=0}=\frac{\left.\frac{\partial^{2} A_{1}}{\partial \theta_{i}^{\prime} \partial \epsilon}\right|_{\theta^{\prime}=\theta} N_{1}^{*}\left(c_{1}-m T_{11}\right)+\left.\frac{\partial^{2} A_{2}}{\partial \theta_{i}^{\prime} \partial \epsilon}\right|_{\theta^{\prime}=\theta} N_{2}^{*}\left(c_{2}-m T_{22}\right)}{1+A_{1} N_{1} T_{11}+A_{2} N_{2} T_{22}}
$$

We have not been able to determine the direction of the dynamics on the IFF, but we studied the behaviour of the system for a range of different values of $c_{i} ; T_{i i} ; a_{i}$ and all different parameter values show that evolution pressure is towards a strong search image when the trade offs are convex and towards no search image when the trade offs are concave. Intuitively, this seems logical, because convex trade offs are relatively worse for intermediate trait values and would therefore indicate more pressure towards extreme trait values (Ma and Levin, 2006).

\subsection{Cost in handling times and non linear trade offs}

The previous results also help us understand what happens when there is both a cost in handling time $(\Delta T \neq 0)$ and non linear trade offs $(z \neq 1)$. In that case we can perform a Taylor expansion with regards to both parameters and the evolution dynamics at IFF can be approximated with:

$$
\nabla W \approx\left(\begin{array}{l}
t_{1}(0)+\left.\Delta T \frac{\mathrm{d} t_{1}(\Delta T)}{\mathrm{d} \Delta T}\right|_{\Delta T=0}+\left.\epsilon \frac{\mathrm{d} t_{1}(\epsilon)}{\mathrm{d} \epsilon}\right|_{\epsilon=0} \\
t_{2}(0)+\left.\Delta T \frac{\mathrm{d} t_{2}(\Delta T)}{\mathrm{d} \Delta T}\right|_{\Delta T=0}+\left.\epsilon \frac{\mathrm{d} t_{2}(\epsilon)}{\mathrm{d} \epsilon}\right|_{\epsilon=0}
\end{array}\right)
$$

such that the system will still quickly evolve towards IFF and the direction will then be determined by both the value of $\Delta T$ and $\epsilon$. When $\Delta T>0$ and $\epsilon>0$ the system will evolve towards less switching, when $\Delta T<0$ and $\epsilon<0$ the system will evolve towards more switching and when $\Delta T>0$ and $\epsilon<0$ or $\Delta T<0$ and $\epsilon>0$ the direction depends on which parameter's influence is greater. 


\section{Discussion}

It is well known that polyphagous predators can form search images for certain prey (Tinbergen, 1960; Allen et al., 1998; Bond and Kamil, 2002). Although the effect of search images on the evolution in prey has been studied (Bond and Kamil, 2006), little has been done on the evolution of a search image in predators. Here we studied a model that allows predators to form search images and models the resulting evolutionary changes. The results showed that selection pressure first leads to a relatively quick convergence to an Ideal Free Foraging (IFF) predator, which is equivalent to the singular strategy for a type II functional response (Rueffler et al., 2006). At the IFF the prey ratio stays constant, while slow evolution leads either to very strong search images when there is a small cost to switching, so that the predators will focus on only one prey and essentially become specialists on the first prey they encounter, or to weaker search images until the predator stops forming search images altogether. At this point our population dynamical model predicts that one of the prey species will go extinct due to predator pressure.

The comparison between a models with and without search images (Schreiber and Tobiason, 2003; Abrams, 2006b,a; Ma and Levin, 2006; Rueffler et al., $2006,2007)$ is interesting. The endpoint for the evolution of the attack rates of a predator which encounters prey in a fine grained fashion, and with a type II functional response but without search image (IFF) is central to our results (Rueffler et al., 2006, 2007). In a model with a search images the search images will evolve quickly towards the IFF. This means that the predator prey system then behaves as if it has a type II functional response and the ratio of prey density will be independent of the strength of the search image. If there is no cost or benefit to prey switching the predator will become an IFF. If there is a cost to switching (which in our model means $\Delta T \neq 0$ or $z \neq 1$ ) this fast evolutionary phase is followed by a period of slow evolution of the search images. If the trade off between the search images is a concave trade off, the search images will slowly disappear due to the fact that there is a benefit to switching (Ma and Levin, 2006). Under a convex trade off the search image will become very strong and the predator will essentially become a specialist that specialises on the first prey it encounters.

The distinction between fast and slow dynamics was shown using a Taylor expansion and these results are, therefore, only proven to be valid for small deviations of the simplest case with equal handling times and linear trade off. However, all numerical work that we did showed that even at large deviations selection pressure towards IFF is high (e.g. see Fig. 2). As a result we are confident that the fast and slow dynamics will still hold even for larger deviations, and we believe that our findings are more general than the mathematical analysis suggests. 
For this model we assumed that predators form a search image after a single encounter. There are indications that this is true for at least some species (Melcer and Chiszar, 1989; Jackson and Li, 2004). Certainly, there will also be predators that only form search images after some time, but this simplification allowed us to solve the system analytically. To be able to model the formation of search images after multiple encounters more needs to be known about how the effects of search images change as a function of number of encounters.

To predict the outcome of evolution it is important to know more about the nature of the trade offs between the conditional attack rates that underly search images. Unfortunately, the only study that measures a trade off directly deals with a different trade off than the one we are interested in (Jessup and Bohannan, 2008). Most studies on trade offs deal only with the fact if a trade off exists or not (for reviews: Palaima, 2007; Jessup and Bohannan, 2008). It will, therefore, be difficult to predict the outcome of the formation of a search image on the evolution of predators in detail. What we have established is that the evolution of search images leads to Ideal Free Foraging and that this is a relatively fast process, which indicates that the IFF should be a common occurrence in predator prey systems irrespective of the trade offs. Once at the IFF the final path towards a specialist or generalist predator is much slower and dependent on the form of the trade off.

The exact cause for the trade off underlying a search image is still under discussion, although Dukas and Kamil (2001) posit that it is caused by limited attention, where a predator is only able to pay attention to one prey type at a time. Another possible explanation would be neural processing bias (Tosh and Ruxton, 2008). But, although the cause is uncertain the existence of the trade off has been shown experimentally (Dawkins, 1971; Pietrewicz and Kamil, 1979; Reid and Shettleworth, 1992; Bond and Kamil, 1999). Faced with this trade off the strength of a predator's search image will influence its fitness. In this manuscript we assumed that the strength of the search image will only be influenced by evolution. Still, it is also possible that behavioural adaptation plays an important role in the strength of this search image. As long as this adaptation is relatively slow, adaptive dynamics can still be used to model its outcome and the presented model can be interpreted as the long term effect of behavioural adaptation (Abrams, 1999; Rueffler et al., 2007).

We would expect these traits often to be coupled, for instance through pleiotropic effects, so that a change in one trait will also cause a change in the other trait. In this case we expect the evolutionary dynamics to be slightly different, but the evolutionary endpoints, and the slow evolutionary dynamics we uncovered, to be similar to the uncorrelated case covered here. Especially since we would expect the traits to be positively correlated, i.e. a stronger search image for one prey will often also cause stronger search images for the other prey, which is in line with our predictions of evolution along the IFF, which also predicts 
a positive correlation between the traits.

A predator with a very strong search image, is a specialist in the sense that it will always keep attacking the prey type it encounters first. This is analogous to an extreme form of food imprinting, where a predator's prey preference is determined by its diet at a young age (Burghardt and Hess, 1966; Punzo, 2002). Imprinting as such could lead to speciation in the predator, because the predator starts to adapt specifically to the prey it is specialising on (see Via, 2001; Berlocher and Feder, 2002, for reviews on this issue). As such the formation of a search image in a predator could lead to different predator species evolving. This would be an important long term effect of the formation of search images by predators.

The results of this model are surprising in that it predicts that the ability to form search images should almost always disappear from populations or become so strong that the predator decides which species to hunt after its first encounter. In contrast, search images have been proven to exist in a large range of predators (Tinbergen, 1960; Pietrewicz and Kamil, 1979; Bond, 1983; Melcer and Chiszar, 1989; Allen et al., 1998; Jackson and Li, 2004; Bond and Kamil, 2006). A possible explanation would be that in real systems predators are unable to completely control the prey densities, due to temporal and spatial heterogeneity in the environment, prey variability, multiple predator species etc. Because selection pressure towards the IFF is much higher one would expect that this result is much more robust and it would be very interesting to see if this prediction would hold in an experimental system.

Search images facilitate polymorphism in prey species due to the protection of rare morphs from extinction (Bond and Kamil, 2002, 2006; Ruxton et al., 2007; Van Leeuwen et al., 2007). Our model predicts that as soon as new mutants appear the predator's search image should quickly adapt towards the IFF, where both prey coexist. If now a new mutant invades again that would mean that the predator would have to adapt to the new mutant as well. This could explain why we find search images in nature, because the predator needs to keep adapting to new morphs and never reach the slow adaptation phase which would lead to the disappearance of search images. This mechanism is similar to the red queen effect, where prey and predator keep evolving, because they are locked in an arms race (Van Valen, 1973).

\section{Acknowledgements}

E.v.L. gratefully acknowledges support by UK Engineering and Physical Sciences Research Council Grant EP/D002249/1 and support by the Netherlands Organisation of Scientific Research (NWO) in the form of Rubicon grant 
825.08 .039

\section{Appendix A Ratio of prey densities at the singular strategy}

Here we derive the ratio of the prey densities at the singular strategy $(\nabla W(\theta)=$ $0)$. First, we solve the partial derivative of the fitness function to mutated trait $\theta_{i}^{\prime}$ and define $f_{i}\left(\theta, N^{*}\right)=h_{i}\left(\theta, N^{*}\right) / k\left(\theta, N^{*}\right)$ resulting in.

$$
\begin{aligned}
& \left.\frac{\partial W\left(\theta^{\prime}, \theta\right)}{\partial \theta_{i}^{\prime}}\right|_{\theta^{\prime}=\theta}= \\
& \frac{c_{1} \frac{\partial h_{1}\left(\theta^{\prime}, N^{*}\right)}{\partial \theta_{i}^{\prime}}+c_{2} \frac{\partial h_{2}\left(\theta^{\prime}, N^{*}\right)}{\partial \theta_{i}^{\prime}}}{k\left(\theta^{\prime}, N^{*}\right)}-\left.\frac{\left(c_{1} h_{1}\left(\theta^{\prime}, N^{*}\right)+c_{2} h_{2}\left(\theta^{\prime}, N^{*}\right)\right) \frac{\partial k\left(\theta^{\prime}, N^{*}\right)}{\partial \theta_{i}^{\prime}}}{k\left(\theta^{\prime}, N^{*}\right)^{2}}\right|_{\theta^{\prime}=\theta}
\end{aligned}
$$

At population dynamical equilibrium the predator's growth is equal to its death rate and we can replace $\left(c_{1} h_{1}\left(\theta, N^{*}\right)+c_{2} h_{2}\left(\theta, N^{*}\right)\right) / k\left(\theta, N^{*}\right)$ with $m$, leading to:

$$
\begin{aligned}
& \left.\frac{\partial W\left(\theta^{\prime}, \theta\right)}{\partial \theta_{i}^{\prime}}\right|_{\theta^{\prime}=\theta}= \\
& \frac{\left.\frac{\partial A_{1}}{\partial \theta_{i}^{\prime}}\right|_{\theta^{\prime}=\theta} N_{1}^{*}\left(c_{1}-m T_{11}\right)+\left.\frac{\partial A_{2}}{\partial \theta_{i}^{\prime}}\right|_{\theta^{\prime}=\theta} N_{2}^{*}\left(c_{2}-m T_{22}\right)}{1+A_{1} N_{1} T_{11}+A_{2} N_{2} T_{22}+\Delta T \frac{a_{21} a_{12} N_{1} N_{2}}{a_{12} N_{1}+a_{21} N_{2}}}+ \\
& \frac{-m N_{1}^{*} N_{2}^{*} \Delta T \frac{\mathrm{d} a_{j i}}{\mathrm{~d} \theta_{i}^{\prime}} \frac{a_{i j}^{2} N_{i}^{*}}{\left(a_{12} N_{1}^{*}+a_{21} N_{2}^{*}\right)^{2}}}{1+A_{1} N_{1} T_{11}+A_{2} N_{2} T_{22}+\Delta T \frac{a_{21} a_{12} N_{1} N_{2}}{a_{12} N_{1}+a_{21} N_{2}}}
\end{aligned}
$$

If $T_{11}+T_{22}=T_{12}+T_{21}$, i.e. $\Delta T=0$ the functional response is identical to a Holling type II functional response for multiple prey species, except that the compound attack rate is dependent on the ratio of prey densities. In this case the partial derivative to $\theta_{i}$ is zero if:

$$
\left.\frac{\partial A_{1}}{\partial \theta_{i}^{\prime}}\right|_{\theta^{\prime}=\theta} N_{1}^{*}\left(c_{1}-m T_{11}\right)=-\left.\frac{\partial A_{2}}{\partial \theta_{i}^{\prime}}\right|_{\theta^{\prime}=\theta} N_{2}^{*}\left(c_{2}-m T_{22}\right)
$$

This can be rewritten to show that at the singular strategy the fraction of prey densities is:

$$
\frac{N_{1}^{*}}{N_{2}^{*}}=-\left.\frac{\partial A_{2} / \partial \theta_{i}^{\prime}}{\partial A_{1} / \partial \theta_{i}^{\prime}}\right|_{\theta^{\prime}=\theta} \frac{\left(c_{2}-m T_{22}\right)}{\left(c_{1}-m T_{11}\right)}
$$




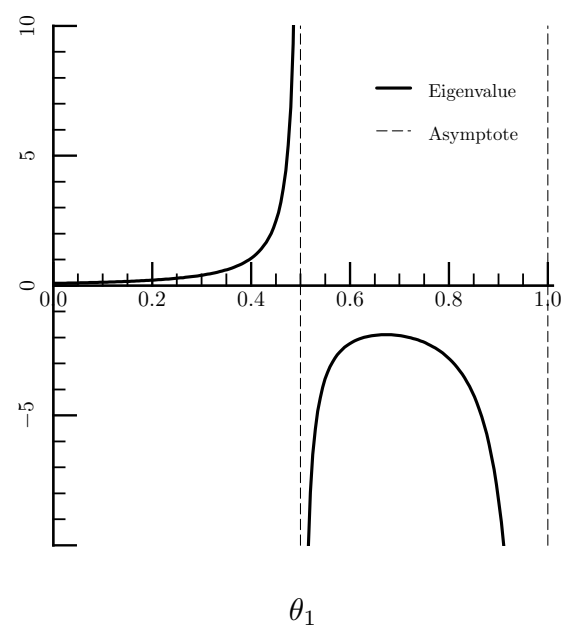

Fig. 3. The eigenvalue of the Jacobian matrix as a function of $\theta_{1}$. There are two asymptotes and between that area the eigenvalue is negative. Parameter values as in Fig. 1

\section{Appendix B Stability analysis IFF}

Defining the canonical equation, allows for a stability analysis around the IFF (Kot, 2001). For this we define the Jacobian matrix at the IFF. Next we were able to find the eigenvalues for this matrix. Since the system exhibits a equilibrium function instead of a single equilibrium point, one of the eigenvalues is zero. The other eigenvalue $(\lambda)$ is relatively complicated, but we were able to find two asymptotes at $\theta_{1}=a_{2} /\left(a_{1}+a_{2}\right)$ and $\theta_{1}=1$, where the denominator of the eigenvalue is zero. The limits around these asymptotes are as follows:

$$
\begin{aligned}
\lim _{\theta_{1} \rightarrow 1} \lambda & =-\infty \\
\lim _{\theta_{1} \rightarrow a_{2} /\left(a_{1}+a_{2}\right)^{+}} \lambda & =-\infty \\
\lim _{\theta_{1} \rightarrow a_{2} /\left(a_{1}+a_{2}\right)^{-}} \lambda & =\infty
\end{aligned}
$$

Solving if this eigenvalue crosses zero for any value of $\theta_{1}$ indicated that there was no real (non complex) trait value for which this eigenvalue is zero. This indicates that between the asymptotes the eigenvalue is always negative and the IFF is stable (Fig. 3).

\section{References}

Abrams, P. A. 1999. The adaptive dynamics of consumer choice. American Naturalist 153:83 - 97 . 
Abrams, P. A. 2006a. Adaptive change in the resource-exploitation traits of a generalist consumer: The evolution and coexistence of generalists and specialists. Evolution 60:427-439.

Abrams, P. A. 2006b. The prerequisites for and likelihood of generalistspecialist coexistence. American Naturalist 167:329-342.

Allen, J. A. 1988. Frequency-dependent selection by predators. Philosophical Transactions of the Royal Society of London Series B-Biological Sciences 319:485 - 503.

Allen, J. A., Raison, H. E., And Weale, M. E. 1998. The influence of density on frequency-dependent selection by wild birds feeding on artificial prey. Proceedings Of The Royal Society Of London Series B-Biological Sciences 265:1031-1035.

Berlocher, S. H. And Feder, J. L. 2002. Sympatric speciation in phytophagous insects: Moving beyond controversy? Annual Review of Entomology 47:773-815.

Bond, A. B. 1983. Visual search and selection of natural stimuli in the pigeon: the attention threshold hypothesis. Journal of Experimental PsychologyAnimal Behavior Processes 9:292 - 306.

Bond, A. B. 2007. The evolution of color polymorphism: Crypticity, searching images, and apostatic selection. Annual Review of Ecology, Evolution, and Systematics 38:489-514.

Bond, A. B. And Kamil, A. C. 1999. Searching image in blue jays: facilitation and interference in sequential priming. Animal Learning $\&$ Behavior 27:461-471.

Bond, A. B. AND KAmiL, A. C. 2002. Visual predators select for crypticity and polymorphism in virtual prey. Nature 415:609 - 613.

Bond, A. B. AND KAmil, A. C. 2006. Spatial heterogeneity, predator cognition, and the evolution of color polymorphism in virtual prey. Proceedings of the National Academy of Sciences of the United States of America 103:3214 -3219 .

Burghardt, G. M. And Hess, E. H. 1966. Food imprinting in the snapping turtle, Chelydra serpentina. Science 151:108-109.

DaWkins, M. 1971. Perceptual changes in chicks: another look at the 'search image' concept. Animal Behaviour 19:566-574.

Dieckmann, U. AND LAW, R. 1996. The dynamical theory of coevolution: A derivation from stochastic ecological processes. Journal Of Mathematical Biology 34:579-612.

DukAs, R. 2002. Behavioural and ecological consequences of limited attention. Philosophical Transactions Of The Royal Society Of Londoneoleol Series B-Biological Sciences 357:1539 - 1547.

Dukas, R. AND KAmiL, A. C. 2001. Limited attention: the constraint underlying search image. Behavioral Ecology 12:192 - 199.

Durinx, M., Metz, J. A. J., And MeszénA, G. 2008. Adaptive dynamics for physiologically structured population models. Journal of Mathematical Biology 56:673-742. 
Fretwell, S. AND LuCAS, H. J. 1970. On territorial behaviour and other factors influencing habitat distribution in birds. Acta Biotheoretica 19:16 36.

Gendron, R. P. 1986. Searching for cryptic prey: Evidence for optimal search rates and the formation of search images in quail. Animal Behaviour 34:898 - 912 .

Geritz, S., Kisdi, E., Meszéna, G., And Metz, J. 2004. Adaptive Speciation, chapter Adaptive Dynamics of Speciation: Ecological Underpinnings. Cambridge University Press.

JACKSON, R. R. AND Li, D. Q. 2004. One-encounter search-image formation by araneophagic spiders. Animal Cognition 7:247 - 254 .

Jessup, C. And Bohannan, B. J. M. 2008. The shape of an ecological trade-off varies with environment. Ecology Letters 11:947-959.

Koт, M. 2001. Elements of Mathematical Ecology. Cambridge University Press.

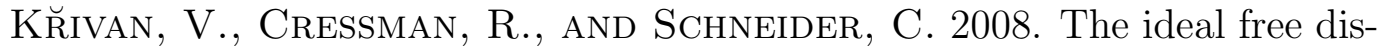
tribution: A review and synthesis of the game theoretic perspective. Theoretical Population Biology 73:403-425.

Levins, R. 1962. Theory of fitness in a heterogeneous environment. i. the fitness set and the adaptive function. American Naturalist 96:361 - 373 .

MA, J. And Levin, S. A. 2006. The evolution of resource adaptation: How generalist and specialist consumers evolve. Bulletin of Mathematical Biology 68:1111-1123.

Melcer, T. And Chiszar, D. 1989. Striking prey creates a specific chemical search image in rattlesnakes. Animal Behaviour 37:477 - 486.

Metz, J. A. J., Nisbet, R. M., And Geritz, S. A. H. 1992. How should we define fitness for general ecological scenarios? Trends in Ecology 86 Evolution 7:198-202.

Murdoch, W. W. 1969. Switching in general predators: Experiments on predator specificity and stability of prey populations. Ecological Monographs $39: 335-354$.

Oaten, A. And Murdoch, W. W. 1975. Switching, functional response and stability in predator-prey systems. American Naturalist 109:299 - 318.

Palaima, A. 2007. The fitness cost of generalization: present limitations and future possible solutions. Biological journal of the linnean society 90:583590.

Pietrewicz, A. T. And Kamil, A. C. 1979. Search image-formation in the blue jay (cyanocitta-cristata). Science 204:1332 - 1333.

Pietrewicz, A. T. And Kamil, A. C. 1981. Foraging behavior: Ecological, ethological, and psychological approaches, chapter Search images and the detection of cryptic prey: An operant approach. New York: Garland.

Punzo, F. 2002. Food imprinting and subsequent prey preference in the lynx spider. Oxyopes salticus (araneae: Oxyopidae). Behavioural Processes 58:177-181.

Reid, P. J. And Shettleworth, S. J. 1992. Detection of cryptic prey: 
search image or search rate? Journal of experimental psychology: animal behavior processes 18:273 - 286.

Rosenzweig, M. L. And MacArthur, R. H. 1963. Graphical representation and stability conditions of predator-prey interactions. American Naturalist 97:209 - 223 .

Rueffler, C., Van Dooren, T. J. M., And Metz, J. A. J. 2006. The evolution of resource specialization through frequency-dependent and frequency-independent mechanisms. American Naturalist 167:81-93.

Rueffler, C., Van Dooren, T. J. M., and Metz, J. A. J. 2007. The interplay between behavior and morphology in the evolutionary dynamics of resource specialization. American Naturalist 169:E34-E52.

Ruxton, G. D., Speed, M. P., And Broom, M. 2007. The importance of initial protection of conspicuous mutants for the coevolution of defense and aposematic signaling of the defense: a modeling study. Evolution 61:21652174.

Schreiber, S. J. And Tobiason, G. A. 2003. The evolution of resource use. Journal of Mathematical Biology 47:56 - 78 .

Tinbergen, N. 1960. The natural control of insects in pine woods. 1. factors influencing the intensity of predation by song birds. Archives de Neerlandais Zoologie 13:265 - 343 .

Tosh, C. R. And Ruxton, G. D. 2008. Body plan of consumed organisms influences ecological range of consumers through neural processing bias. American Naturalist 171:267-273.

Van Leeuwen, E., Jansen, V. A. A., and Bright, P. W. 2007. How population dynamics shape the functional response in a one-predator-twoprey system. Ecology 88:1571 - 1581.

VAn Valen, L. 1973. A new evolutionary law. Evolutionary Theory 1:1-30.

VIA, S. 2001. Sympatric speciation in animals: the ugly duckling grows upon. Trends in Ecology \& Evolution 16:381-390. 